\title{
Interação Universidade-Empresa no Brasil: Evidências do Diretório dos Grupos de Pesquisa do CNPq
}

\author{
- MÁrcia SiqueIRA RAPINI*
}

\begin{abstract}
RESUMO
O presente trabalho investiga a interação universidade-empresa no Brasil por meio de informações disponíveis no Diretório dos Grupos de Pesquisa do CNPq coletadas no Censo 2002. Apesar dos problemas inerentes à base de dados, estas informações fornecem um panorama recente da interação entre universidades e empresas no País. Os resultados encontrados apontam que as relações de colaboração são concentradas em termos de áreas do conhecimento dos grupos de pesquisa, instituições às quais os grupos estão vinculados e setor de atividade das empresas. As interações mais freqüentes entre os agentes foram as atividades de Engenharia não-rotineira, de Consultoria Técnica e Treinamento de pessoal. À medida que estes resultados forem ao encontro das especificidades do Sistema Nacional de Inovação presente no País, incentivos podem ser dados com vistas a aumentar a transferência do conhecimento acadêmico para as empresas.
\end{abstract}

\section{PALAVRAS-CHAVE}

interação universidade-empresa, grupos de pesquisa, área do conhecimento, setor de atividade

\begin{abstract}
The present work investigates university-industry interactions in Brazil through available data from CNPq's Research Groups Directory collected in Census 2002. Although limitations intrinsic to information collected, the database supplies some evidence from recent university-industry interactions in Brazil. Collaborative relations are restricting to some members, and the agents involved are concentrated in both sides. The more frequent relationships were no routine engineering, consultancy and trainament. This result were in accordance with Brazilian's NSI, but also suggests that's much more can be done fostering academic knowledge transference to firms in the country.
\end{abstract}

KEY WORDS

university-industry interactions, research groups, knowledge area, sector of activity

\author{
JEL CLASSIFICATION \\ O30, 039
}

\footnotetext{
* Mestre em Economia no IE-UFRJ. Pesquisadora do Grupo de Economia da Ciência e da Tecnologia do CEDEPLAR/ UFMG e IEL/FIEMG. E-mail: msrapini@cedeplar.ufmg.br. End. para correspondência: Rua Bambuí, $752 / 204$ - Anchieta - Cep: 30310-320 - Belo Horizonte - MG.

(Recebido em março de 2005. Aceito para publicação em julho de 2006).
} 


\section{INTRODUÇÃA}

O processo de inovação, talvez mais do que qualquer outra atividade econômica, depende do conhecimento. (Feldman, 1994). O novo papel da informação e do conhecimento nas economias e no processo produtivo tem levado a um reposicionamento do papel desempenhado pelas universidades, as quais não apenas são responsáveis pelo treinamento, como passaram a fornecer conhecimento crucial para a evolução de alguns setores industriais.

A intensificação das interações entre universidades e empresas, a partir dos anos 1980, se por um lado vem se refletindo na criação de diversos novos mecanismos institucionais de transmissão de tecnologia e de conhecimento, por outro sinaliza um processo caracterizado por fluxos bilaterais de conhecimentos e técnicas. (Meyer-Kramer e Schmoch, 1998). A estas mudanças acrescenta-se a presença de institucionalidades e de uma forte articulação com a infra-estrutura de ciência e tecnologia nacional (Pavitt, 1998), de modo que o entendimento deste processo não pode ser dar dissociado do desenvolvimento e consolidação do Sistema Nacional de Inovação (SNI).

A literatura que investiga interação universidade-indústria é empírica, baseada em três fontes de informação: estudos de casos, análises de patentes e bibliométricas ou em grandes pesquisas/levantamentos. Este trabalho investiga este tema com base nas informações disponíveis no Diretório dos Grupos de Pesquisa do CNPq, referentes ao ano de 2002, ano-base para o qual as informaçôes foram solicitadas. A metodologia para a avaliação destes dados buscou ajustar o questionário aos objetivos do trabalho. Esta base de dados, pouco explorada, apesar de não contar com o nível de detalhamento de estudos empíricos, abrange um número maior de instituições em várias localidades do País, sendo, além disso, informações recentes.

Além desta introdução, o trabalho conta com mais quatro partes, sendo a primeira uma revisão da literatura referente à interação universidade-indústria, contemplando também especificidades destas articulações nos países em desenvolvimento. A segunda parte traz um resumo das relações entre universidades e empresas no País, vinculando-as aos processos de industrialização e de consolidação da infra-estrutura nacional de C\&T. A terceira parte examina a interação dos grupos de pesquisa do CNPq com empresas, por tipos de relacionamento e grande área do conhecimento, além de explorar particularidades de articulações entre área do conhecimento e setor de atividade. A última parte conclui o trabalho apresentando os principais resultados. 


\section{INTERAÇÃO UNIVERSIDADE-INDÚSTRIA: REVISÃO DA LITERATURA}

O aumento da contribuição do conhecimento científico ao processo tecnológico remete a um importante papel desempenhado pelas universidades na medida em estas que permanecem como fonte primordial de geração deste conhecimento.

As contribuições das universidades para o processo de inovação nas firmas podem ser sintetizadas como: fonte de conhecimento de caráter mais geral necessários para as atividades de pesquisa básica (Nelson, 1990); fonte de conhecimento especializado relacionado à área tecnológica da firma (Klevorick et al., 1995); formação e treinamento de engenheiros e cientistas capazes de lidar com problemas associados ao processo inovador nas firmas (Rosenberg e Nelson, 1994; Pavitt, 1998); criação de novos instrumentos e de técnicas científicas (Rosenberg, 1992); criação de firmas nascentes (spin-offs) por pessoal acadêmico (Stankiewics, 1994; Etzkowitz, 1999).

A amplitude e intensidade das interaçóes universidade-empresa, por parte das firmas, são fortemente associadas às oportunidades tecnológicas e ao grau de apropriabilidade enfrentados pelo setor de atividade. (Klevorick et al., 1995). Nas universidades, varia de acordo com as áreas do conhecimento (Saltin e Martin, 2001), de forma que a contribuição da ciência ao processo inovador é compelida por especificidades setoriais. Da caracterização pioneira de Pavitt (1984) aos setores baseados na ciência (sciencebased), que seriam aqueles nos quais as inovações estariam diretamente relacionadas aos avanços no conhecimento científico, acrescentam-se trabalhos posteriores, baseados em metodologias distintas, ${ }^{1}$ e voltados para a identificação da interação entre as descobertas científicas e o avanço tecnológico nas firmas.

Essas investigações revelaram que os setores de atividade com interações mais intensas com a ciência são os que incluem áreas tecnológicas relacionadas à engenharia genética, química orgânica e inorgânica, tecnologia de alimentos, biotecnologia, tecnologia a laser, e microeletrônica (telecomunicações, componentes eletrônicos, processamento de dados). Os setores vinculados às indústrias são os da química, petroquímica, farmacêutica, de semicondutores, computadores, instrumentos eletrônicos, equipamentos elétricos e aeroespacial, sendo as áreas científicas as de maior dispersão ou aplicabilidade setorial: ciência da computação, ciência dos materiais, química, informática, metalurgia, física e matemática. (Klevorick et al.,1995; Cohen et al., 2002; Schartinger et al.,2002; Godin, 1996; Mansfield, 1991; Grupp, 1996).

1 Dentre as metodologias utilizadas têm-se: investigação dos artigos científicos citados nas patentes - non patent references (Narin et al., 1997; Grupp, 1996); investigação da área científica de artigos publicados por firmas (Godin, 1996); e investigação via estudos, pesquisas e ou questionários aplicados às firmas (Klevorick et al., 1995; Mansfield, 1991; Cohen et al., 2002) e a pesquisadores acadêmicos (MeyerKramer e Schmoch, 1998; Schartinger et al., 2001 e 2002). 
Além das especificidades setoriais, a intensidade da interação universidade-empresa é compelida por outros fatores, como os referentes ao setor industrial (tamanho da firma e características do desenvolvimento de novos produtos), ao setor de pesquisa público (políticas, disponibilidade de expertise, papel do mesmo como usuário), à tecnologia (características gerais, estágio de desenvolvimento, dinamismo da área), à firma (existência de base de conhecimento, propensão à interação ${ }^{2}$ ). (Faulkner e Senker, 1994). Estes fatores, além de influenciar a intensidade das interações universidade-indústria, conferem à mesma um caráter fortemente path dependency, que enfrenta problemas de adoção de novos paradigmas tecnológicos e trajetórias tecnológicas de forma suficientemente rápida. (Meyer- Kramer e Schmoch, 1998, p. 845).

Outro fator amplamente investigado na literatura é a importância da proximidade geográfica na interação universidade-indústria. Os trabalhos realizados visaram identificar os efeitos de 'transbordamento' (spill-overs) do componente tácito do conhecimento científico gerado nas universidades para as atividades de P\&D industrial (Jaffe et al., 1993; Acts et al., 1994), a importância de colaborações informais e contatos cara a cara em regiões de alta tecnologia (Varga, 1997; Mansfield e Lee, 1996) e a contribuição da infra-estrutura de pesquisa no desenvolvimento de capacidades inovadoras em regiões (Dorfman, 1983; Feldman, 1994). As contribuições, contudo, variam de acordo com o tamanho da firma e intensidade de P\&D (Beise e Stalh, 1999), nível de formalização das interações (Audretsch e Stephan, 1996) e a presença de institucionalidades locais (Breschi e Lissoni, 2001).

Nos países em desenvolvimento, uma primeira distinção em se tratando de interação universidade-indústria reside no baixo nível de atividades de P\&D desenvolvidas pelas firmas. Conseqüentemente, com raras exceções, as firmas não têm como rotinas e estratégia de concorrência e crescimento a geração interna de conhecimento. A maior parte das atividades de P\&D é realizada pelo setor público, via empresas estatais, instituições de pesquisa e universidades federais. (Sutz, 2000).

A fraca demanda por conhecimento, em termos quantitativos e qualitativos, por parte das firmas, é manifestada no pouco interesse em estabelecer relaçóes com universidades, sendo que a contribuição mais expressiva destas últimas reside na formação de recursos humanos. As interações, quando presentes, limitam-se a atividades de consultoria, serviços de rotina (mensuração, testes e controle de qualidade) e não a pesquisas de alto nível e de desenvolvimento experimental. Em termos da complexidade do papel desempenhado pelas universidades na periferia, estas, em vez de produtoras de conhe-

2 Importante fator a ser considerado é a origem do capital da empresa, uma vez que a presença de empresas multinacionais e de capital estrangeiro em setores industriais relevantes ou orientados internacionalmente compromete o processo de troca de conhecimento por meio da interação U-I, tendendo a ocorrer com o exterior, como identificado por McFetridge (1993) no Canadá e por Schartinger et al. (2002) na Áustria. 
cimento, são apenas praticantes do conhecimento de fronteira existente, definindo um perfil muito mais de consultoria do que de pesquisa em relação à indústria. ${ }^{3}$

Dentre os problemas recorrentes em termos das relações de cooperação entre universidades e empresas nos países em desenvolvimento destacam-se a ausência de mecanismos eficazes na definição dos direitos de propriedade, dificuldades de comunicação, burocracia, inadequação do pessoal de pesquisa, financiamento adequado, fatores socioculturais e diferenças de cultura da universidade e indústria em termos de atividades de P\&D relacionados ao curto versus longo prazo. (Jasinski, 1997; Oyebisi et al., 1996). Em relação às diferenças culturais, salienta-se a distinta trajetória de institucionalização da atividade científica e dos cientistas nos países em desenvolvimento, que na ausência de padrões endógenos de legitimação buscaram a afirmação como comunidade em circuitos internacionais (Sutz, 1997), alienando-se dos problemas sociais e tecnológicos do País. (Velho, 1996).

Como síntese, pode-se valer do sistematizado por Sutz (2000) na observação de algumas experiências de articulação universidade-indústria-governo em países da América Latina: (a) o envolvimento das firmas abaixo das expectativas, tanto em termos quantitativos como qualitativos; (b) ausência de 'conhecimento relevante' na solução de problemas quando a demanda existe; (c) pouca alteração no comportamento geral das firmas no que diz respeito ao relacionamento com universidades. O mau emparelhamento da interação universidade-indústria é explicado pela autora como sendo resultante da designação de mecanismos não adequados ou da utilização insuficiente dos mecanismos existentes.

\section{INTERAÇÃO UNIVERSIDADE - INDÚSTRIA NO BRASIL}

O Brasil, até 1945, se caracterizou pela inexistência de um projeto de industrialização definido. Quando este aparece, a política industrial não vislumbra como essencial o incentivo à ciência e tecnologia, provocando um descompasso entre as políticas de desenvolvimento industrial e de desenvolvimento científico e tecnológico. (Cassiolato et al., 1996). O esforço de construção institucional entre 1930 e 1970, apesar de ter possibilitado a convergência da base industrial e tecnológica nacional a patamares internacionais, não foi funcional para o alcance das novas tecnologias. (Suzigan e Villela, 1997, p. 31). A desconexão entre a infra-estrutura de C\&T e o setor produtivo se agrava nos anos 1980 e 1990 com a crise financeira do Estado, que se reflete em

3 Arocena e Sutz (2003) propõem a denominação das universidades na periferia de 'universidade isolada consultora' em contrapartida a 'universidade conectada empreendedora' no centro, que além de mais articuladas com as firmas, estão envolvidas na produção de conhecimento de fronteira de potencial aplicabilidade ao setor produtivo. 
um sistemático processo de erosão da primeira, comprometendo não apenas a formação de recursos humanos, mas também a habilidade da mesma em corresponder à capacidade produtiva do País. (Suzigan e Villela, 1997, p. 130).

Até os anos 1970, os programas governamentais voltados à redução da dependência tecnológica externa acabaram por privilegiar a entrada da tecnologia estrangeira e o estabelecimento de multinacionais nos setores mais sofisticados. Neste cenário, a comunidade científica, por sua vez, limitou-se a aplicar os recursos disponíveis no aumento de seu desempenho a níveis internacionais, sendo a opção pela pesquisa básica uma condição de sobrevivência. (Velho, 1996). O fracasso da conexão entre a comunidade empresarial e a acadêmica levou o Estado a "transformar ele mesmo num demandante de pesquisa e tecnologia." (Velho, 1996, p. 49). ${ }^{4}$

Uma primeira iniciativa partindo da marinha esteve relacionada ao design de hardware do primeiro microcomputador nacional envolvendo a Universidade de São Paulo (USP) e a Pontifícia Universidade Católica do Rio de Janeiro, em 1971. Posteriormente, em 1973, foi criada a Digibrás, holding estatal, que visava à promoção da indústria brasileira de computadores. Pela primeira vez os esforços de desenvolvimento de tecnologia local eram articulados com firmas de capital nacional.

Similarmente, em 1976, o Centro de Pesquisas e Desenvolvimento da Telebrás (CPqD) foi estabelecido próximo à UNICAMP, tendo a escolha sido motivada pela infra-estrutura educacional e de pesquisa existente na universidade. Os objetivos do CPqD eram o desenvolvimento de estratégias para o setor de telecomunicações no País, sendo uma junção de esforços das universidades locais, dos produtores de equipamentos de telecomunicações, outras instituiçôes da região, assim como o desenvolvimento de mão-de-obra qualificada. (Szapiro, 2003). Da mesma forma, a existência do Centro de Tecnologia da Aeronáutica (CTA) e do Instituto Nacional de Pesquisas Espaciais (INPE), em São José dos Campos, propiciou as condições necessárias para a criação da Embraer em 1969, empresa estatal destinada à fabricação de aviões. Pelo lado do empresariado, observa-se a iniciativa da Confederação Nacional da Indústria (CNI), que criou o Instituto Euvaldo Lodi (IEL) em 1969, com a finalidade de promover a interação universidade-indústria. (Rappel, 1999, p. 96).

Nos anos 1980, o I Plano de Desenvolvimento Nacional da Nova República (1986-89) reconheceu que até aquele momento a oferta de tecnologia tinha sido freqüentemente baseada em critérios acadêmicos, sem considerações significativas às necessidades da base industrial e do mercado. (Dahlman e Frischtak, 1993, p. 435). A partir de então,

4 De acordo com Velho (1996), a opção dos cientistas por desenvolverem atividades científicas não vinculadas às necessidades sociais ou tecnológicas do País era a estratégia política de não-cooptação com regime autoritário vigente. Da mesma forma, relações com as classes empresariais, aliadas do Estado autoritário, representavam elos com o regime. 
tem-se a primeira iniciativa de aproximação entre a academia e a indústria partindo do governo federal com o 'Programa de Inovação Tecnológica' no âmbito do Conselho Nacional de Desenvolvimento Científico e Tecnológico (CNPq). Posteriormente, o 'Programa de Implantação de Parques Tecnológicos' possibilitou a criação dos primeiros parques tecnológicos e das incubadoras de empresas no País. Em 1987, a Associação Nacional de Entidades Promotoras de Empreendimentos de Tecnologias Avançadas (ANPROTEC) foi concebida, o que significou avanços em termos de coordenação. (Theis, 2002).

A ênfase na expansão da infra-estrutura de C\&T, principalmente no desenvolvimento de recursos humanos, refletiu as preocupações anteriores. O 'Programa de Capacitação de Recursos Humanos para Áreas Estratégicas' (RHAE), instituído em 1987, sintetizava "a percepção do Estado de que havia lacunas entre o conbecimento gerado na universidade e sua transferência para o setor produtivo. Havia, portanto, a necessidade de estabelecer um link entre academia e indristria." (Costa, 1999, p. 278). Os investimentos anteriores no acréscimo de bolsas propiciaram o sucesso do programa, sendo que entre 1985 e 1990 o crescimento das bolsas de mestrado foi de 83,4\%, de doutorado $111,09 \%$ e de iniciação científica $233 \%$.

Nos anos 1990, a Política Industrial e de Comércio Exterior concebida também atribuiu significativa importância à associação entre universidades e empresas na modernização tecnológica do parque industrial nacional e no aumento da participação do setor privado nos investimentos de C\&T. (Velho, 1996, p. 55). No âmbito federal, foram criados programas especiais tendo como objeto a pesquisa aplicada e o desenvolvimento experimental nas áreas de engenharias. (Rappel, 1999, p. 101). O maior deles foi uma ação conjunta dos Ministérios de Ciência e Tecnologia e da Educação, que criou o programa de Redes Cooperativas de Pesquisa (RECOPE) para estimular e apoiar a constituição de redes de instituições de pesquisa e empresas em torno de projetos cooperativos. Na esfera do III Programa de Apoio ao Desenvolvimento Científico e Tecnológico (PADCT), o Projeto Plataforma foi criado para favorecer a integração dos esforços entre universidades, institutos de pesquisa e empresas industriais na geração de projetos cooperativos. ${ }^{5}$

Adicionalmente a estes programas foram concebidos incentivos fiscais às empresas com vistas a aumentar a capacitação e a competitividade, por meio das Leis $\mathrm{n}^{\circ} 8.248$ de 1991 e 8.661 de 1993. A primeira lei estabeleceu que as empresas nas áreas de informática e automação deveriam investir $5 \%$ do seu faturamento em P\&D, e dentre estes pelo menos $2 \%$ deveriam, obrigatoriamente, ser aplicados em convênios

5 Dentre os 10 programas apoiados pelo PADCT em 1998, destaca-se o Programa de Automação Industrial na Bahia, envolvendo 8 indústrias petroquímicas e 2 universidades, tendo gerado 7 projetos cooperados. 
com universidades ou instituições de pesquisa. A segunda lei dispunha-se a conceder incentivos fiscais para a capacitação tecnológica das empresas dos setores industriais e agropecuário que executassem, respectivamente, o Programa de Desenvolvimento Tecnológico Industrial (PDTI) e o Programa de Desenvolvimento Tecnológico Agropecuário (PDTA). De acordo com Silva e Mazzali (2001), este conjunto de incentivos, até setembro de 1995, beneficiou 27 programas de desenvolvimento tecnológico, referentes a 26 empresas isoladas e a um consórcio abrangendo 40 empresas. Estas medidas, contudo, tornaram-se inoperantes com a publicação da Lei 9532, de 1997, que reduziu para 4\% a dedução do imposto de renda para os investimentos em tecnologia. (Cassiolato et al., 1996).

Em nível estadual, observa-se também a criação de mecanismos específicos de estímulo à cooperação da comunidade acadêmica com o setor empresarial provenientes das Fundações de Amparo à Pesquisa. Como exemplo, vale enumerar a FAPERG, que em 1989 lançou o primeiro edital de projetos de pesquisa que assegurassem a integração universidade e empresa (Frantz, 1999), tendo até 1998 financiados cerca de 200 projetos. (Vargas et al., 1999). A FAPESP vem criando "mecanismos para intensificar a disseminação do conhecimento, tornando-o mais acessivel à empresa” (Cruz, 1999, p. 235) com a criação de Programas como o de Parceria para Inovação Tecnológica (PITE) e o de Inovação na Pequena Empresa (PIPE).

Do lado das empresas, têm-se iniciativas recentes dos Institutos Euvaldo Lodi, com a criação dos Fóruns de Tecnologia (ForumTec) no Ceará (em 1996) e na Bahia (em 1997) "cujo objetivo é articular as instituições que compõem o Sistema Estadual de Ciência e Tecnologia, promovendo a geração de projetos cooperativos que captem recursos para o desenvolvimento tecnológico local.” (Rappel, 1999, p. 102). A Associação Nacional de Pesquisa e Desenvolvimento das Empresas Industriais (ANPEI), criada em 1984, também vem, mais recentemente, realizando iniciativas na aproximação entre a indústria e a universidade.

A investigação de estudos de casos revela conexões parciais, construídas historicamente, como é o caso da Petrobrás, com seis Centros de Excelência atualmente, da EMBRAPA e do IPT. Por parte das universidades, relatos de interação e de relativo sucesso na UNICAMP (Brisolla et al., 1997), UFSCAR e USP (Gregolin, 1999) fazem também referência às articulaçóes construídas ao longo do tempo, muitas vezes estando estas dentre as missões iniciais da instituição. Além destes casos, o crescente comprometimento de universidades no desenvolvimento local e regional observado recentemente é atribuído muito mais à subordinação a políticas estaduais específicas de C\&T do que a iniciativas partidas de instituiçóes ou de empresas, como bem ilustra o Programa Regional de Cooperação Científica e Tecnológica (PRCT) do noroeste do Rio Grande/RS. (Schneider, 1999). 
Dentre os problemas identificados em estudos de casos, que refletem as ineficiências e fragilidades das interações recentes entre universidades e empresas no País, estão: o baixo conteúdo científico e curto prazo requerido para as soluções industriais que não estimulam os contratantes a investir em ciência e tecnologia (Castro e Balán, 1994, apud Brisolla et al., 1997); a ausência de interlocutores adequados nas firmas dificultando a comunicação (Brisolla et al., 1997); setor produtivo pouco inovador (Melo, 1999); ausência de instrumentos adequados nas universidades para a comercialização de tecnologia (Hemais et al., 2000); e a pouca flexibilidade das instituiçôes de ciência e tecnologia (Salomão, 1999).

Este cenário é confirmado pela atual configuração industrial nacional não voltada para a geração interna de tecnologia própria, como constatado pela PINTEC (2000). A pesquisa realizada pelo Instituto Brasileiro de Geografia e Estatística (IBGE) revelou baixa taxa de inovação nacional (em média 32), reflexo de poucos investimentos em atividades de P\&D por parte do setor privado. A principal fonte de incorporação e desenvolvimento de novas tecnologias industriais deu-se via aquisição de máquinas e equipamentos, em um contexto no qual nas empresas a maior proporção é de atividades de P\&D ocasionais (57,13\% do total). Confirmando tal assertiva, as principais fontes de informação para a implementação de inovações foram as oriundas de interações de natureza comercial (com fornecedores, clientes, consumidores e empresas concorrentes), em detrimento de fontes de informações institucionais, como as provenientes de universidades e instituições de pesquisa.

Na mesma direção, a cooperação com as demais organizações na implementação de atividades inovadoras foi pouco utilizada pelas firmas industriais no Brasil. Do conjunto das firmas inovadoras, somente 11\% valeram-se deste tipo de estratégia. Em termos de parceiros, a maior proporção de relações de cooperação ocorreu com fornecedores e clientes, respectivamente 6\% e 5\% das empresas inovadoras, e apenas $3,7 \%$ com universidades. ${ }^{6}$ Enquanto que a supremacia dos agentes da cadeia produtiva como fontes de informação relativamente às demais vai ao encontro do identificado na literatura (Cohen et al, 2002), a pouca utilização das universidades é um contraste, visto que nos países da OCDE o porcentual de colaboração está em torno de 10\%. (IBGE, 2003).

Uma investigação intersetorial revela que além das especificidades setoriais onde uma maior proximidade entre descobertas científicas e inovações está presente, a cooperação com universidades no País vincula-se significativamente à presença de atividades internas de $\mathrm{P} \& \mathrm{D},{ }^{7}$ sinalizando que 'alguma linguagem em comum' é necessária para

6 Alto e médio grau de importância atribuído a esta opção no questionário.

7 Conforme levantado na literatura. (Rosenberg, 1990; Pavitt, 1991). 
o estabelecimento de interações. É à luz destas especificidades que a próxima seção deve ser lida.

\section{INTERAÇÃO DOS GRUPOS DE PESQUISA DO CNPq COM EMPRESAS}

O Diretório dos Grupos de Pesquisa do CNPq reúne informações sobre os grupos de pesquisa em atividade no País abrangendo pesquisadores, estudantes, técnicos, linhas de pesquisa em andamento, produção científica, tecnológica e artística geradas pelos grupos. Apesar de ser caracterizado por uma base de informações de preenchimento opcional, o universo abrangido pela mesma vem aumentando ao longo do tempo, podendo-se supor ter relativa representatividade da comunidade científica nacional. (Carneiro e Lourenço, 2003).

As informaçốes exploradas ao longo desta seção foram obtidas, mediante consultas on-line, do Censo 2002 do Diretório dos Grupos de Pesquisa do CNPq no módulo 'Plano Tabular', sendo exceção os dados examinados na seção 3.1, requisitados, mediante tabulação especial, ao CNPq. Este módulo disponibiliza o conjunto de variáveis que podem ser agregadas, de maneiras diversas, na construção de tabelas. A escolha das variáveis a serem analisadas visou obter resultados comparáveis aos já existentes na literatura. A versão 4.0 do Diretório, objeto de investigação do trabalho, abrange 268 instituições, com 15.158 grupos de pesquisa cadastrados.

Dentre esses, 1.279 grupos de pesquisa, equivalentes a $8,4 \%$ do total, relataram algum tipo de relacionamento com empresas, pertencentes a 159 instituições cadastradas no Diretório. A Tabela 1 apresenta o número de grupos de pesquisa que constaram de relações de colaboração com empresas e a soma dos relacionamentos declarados em cada grande área do conhecimento. Observa-se que duas grandes áreas do conhecimento concentram mais de $60 \%$ do total dos relacionamentos dos grupos de pesquisa com empresa, quais sejam: Engenharias e Ciência da Computação $(43,8 \%)$ e Ciências Agrárias (19,5\%). O primeiro caso é de alguma forma esperado, visto abranger áreas de tradicional proximidade com as práticas industriais. O segundo, por sua vez, reflete a especialização nacional em agroindústria, especificidades da difusão da tecnologia e incentivos públicos de longo prazo para o desenvolvimento da agricultura desde 1960.

Há também significativas interações dos grupos de pesquisa nas Grandes Áreas de Ciências Exatas e da Terra e Ciências Biológicas, com respectivamente 10,3\% e 9,7\% do total dos relacionamentos. A participação dos relacionamentos associados aos grupos de Humanidades (Ciências Sociais e Aplicadas, Ciências Humanas e Lingüística, Letras e Artes) e de Ciências da Saúde é inferior a 6\%. Este resultado para as áreas de 
Humanidades é de alguma forma esperado, mesmo que a literatura esteja apontando para uma maior interação com empresas, principalmente das do setor de serviços. (Schartinger et al., 2002). No entanto, para as de Ciências da Saúde tal panorama é preocupante, remetendo a especificidades nacionais. (Cassiolato e Albuquerque, 2000)..$^{8}$

TABELA 1 - TOTAL DE GRUPOS DE PESQUISA COM RELACIONAMENTOS E SOMA DOS RELACIONAMENTOS DECLARADOS POR GRANDE ÁREA DO CONHECIMENTO, BR ASIL, 2002

\begin{tabular}{l|rr|rr}
\hline Grande Área do Conhecimento & \multicolumn{2}{|c|}{$\begin{array}{c}\text { Grupos de pesquisa com } \\
\text { relacionamento } \\
(1)\end{array}$} & \multicolumn{2}{c}{$\begin{array}{c}\text { Soma dos } \\
\text { relacionamentos } \\
(2)\end{array}$} \\
\hline Ciências Agrárias & 274 & $21,4 \%$ & 1640 & $19,5 \%$ \\
Ciências Biológicas & 119 & $9,3 \%$ & 819 & $9,7 \%$ \\
Ciências Exatas e da Terra & 162 & $12.7 \%$ & 864 & $10,3 \%$ \\
Ciências Humanas & 59 & $4,6 \%$ & 454 & $5,4 \%$ \\
Ciências Sociais Aplicadas & 75 & $5,9 \%$ & 402 & $4,8 \%$ \\
Ciências da Saúde & 116 & $9,1 \%$ & 489 & $5,8 \%$ \\
Engenharias e Ciência da Computação & 460 & $36,0 \%$ & 3688 & $43,8 \%$ \\
Lingüística, Letras e Artes & 14 & $1,1 \%$ & 61 & $0,7 \%$ \\
Total & 1279 & $100,0 \%$ & 8417 & $100,0 \%$ \\
\hline
\end{tabular}

Fonte: Diretório dos Grupos de Pesquisa do CNPq, Censo 2002, elaboração própria.

A informação referente aos tipos de relacionamentos possíveis entre grupos de pesquisa e empresas foi fornecida pelos líderes dos grupos. Cada líder do grupo pode atribuir até três tipos de relacionamento mais freqüente com empresas, tipos esses que se encontram listados no Quadro 1. O primeiro bloco da tabela sintetiza os relacionamentos possíveis oriundos dos grupos de pesquisa para empresas, ao passo que o segundo resume os relacionamentos possíveis provenientes das empresas para os grupos de pesquisa. Dentre estes, os de número 4 e 12 foram excluídos das investigações subseqüentes por não estarem necessariamente associados a relações de interesse mútuo. ${ }^{9}$

As informações disponibilizadas não permitem identificar o grau de relevância ou importância atribuída a cada tipo relacionamento, de forma que análises similares às realizadas na literatura (Meyer-Kramer e Schmoch, 1999; Klevorick et al., 1996; Cohen

8 Segundo os autores, o progresso tecnológico no setor Saúde no Brasil se caracteriza por um "processo de absorção passiva e desordenada” (p. 67), sendo pouco expressivas as interações da academia com o setor farmacêutico.

9 Estes relacionamentos equivalem a $18 \%$ do total. 
et al., 2002) não são possíveis de realizar. As análises são primordialmente baseadas na soma dos distintos tipos de relacionamentos em cada grande área do conhecimento e na participação relativa dos mesmos em cada conjunto de investigação. Ademais, limitações e problemas inerentes à coleta dos dados, decorrentes da subjetividade das percepções individuais, não estão sob controle. ${ }^{10}$

\section{QUADRO 1 - TIPOS DE RELACIONAMENTO DOS GRUPOS DE PESQUISA COM EMPRESAS DE ACORDO COM O FLUXO DE ORIGEM, BRASIL, 2002}

\begin{tabular}{|c|l|}
\hline & Provenientes dos Grupos de Pesquisa para as Empresas (Grupos de Pesquisa-> Empresas) \\
\hline 1 & Atividade de consultoria técnica não contempladas nos demais tipos \\
3 & Atividades de engenharia não rotineira, inclusive o desenvolvimento de protótipo cabeça de série ou planta piloto \\
4 & Fornecimento de insumos materiais para as atividades sem vinculação a um projeto específico de interesse mútuo \\
5 & Pesquisa científica com considerações de uso imediato dos resultados \\
6 & Pesquisa científica sem considerações de uso imediato dos resultados \\
7 & Transferência de tecnologia \\
8 & Treinamento do pessoal incluindo cursos e treinamento em "serviço" \\
9 & Outros tipos predominantes de relacionamento que não se enquadrem em nenhum dos anteriores \\
\hline 10 & Provenientes das Empresas para os Grupos de Pesquisa (Empresas-> Grupos de Pesquisa) \\
\hline 11 & Desenvolvimento de software não rotineiro \\
12 & Fornecimento de insumos materiais para as atividades sem vinculação a um projeto específico de interesse mútuo \\
13 & Transferência de tecnologia \\
14 & Treinamento do pessoal incluindo cursos e treinamento em "serviço" \\
\hline
\end{tabular}

Fonte: Diretório dos Grupos de Pesquisa - CNPq.

A Tabela 2 sintetiza a freqüência dos tipos de relacionamentos descritos no Quadro 1 em cada grande área do conhecimento. O primeiro bloco traz os tipos de relacionamentos oriundos dos grupos de pesquisa tendo como destino as empresas. Estes representam cerca de $76 \%$ do total de relacionamentos, sendo que nesta base de dados as interações de colaboração de interesse mútuo partem primordialmente das universidades e instituições públicas de pesquisa. ${ }^{11}$ A maior parcela de colaboração oriunda das empresas para os grupos de pesquisa (segundo bloco) ocorre em Engenharias e Ciência da Computação, abrangendo 13\% do total.

10 Como exemplo do problema das distintas percepções dos indivíduos em relação ao tema transferência de tecnologia ver Moravcsik (1983, p. 288).

11 A identificação da natureza das instituições envolvidas encontra-se em Rapini (2004). 


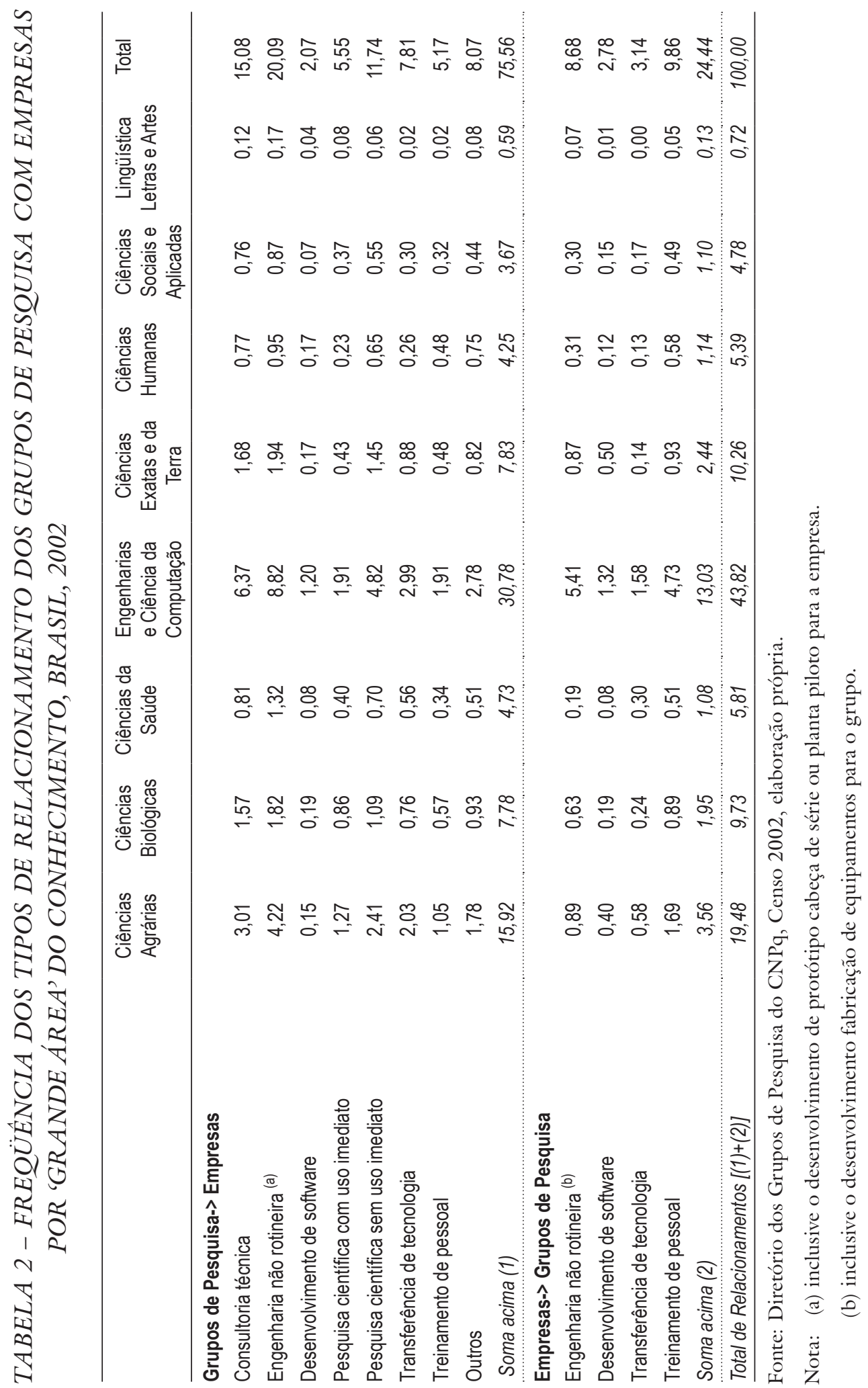


Dentre os relacionamentos provenientes dos grupos de pesquisas para as empresas (primeiro bloco), os de maior frequiência são, respectivamente, as atividades de 'Engenharia não-rotineiras', com $20 \%$ do total, 'Consultoria Técnica', com $15 \%$, e 'Pesquisa científica sem considerações de uso imediato dos resultados', com aproximadamente $12 \%$ do total. As atividades de 'Engenharia não rotineira', por incluírem o desenvolvimento de protótipo ou planta-piloto para as empresas, estariam contemplando atividades associadas às etapas iniciais e de definição de projetos. Comparativamente aos resultados encontrados nos EUA por Cohen et al. (2000), em que a maior freqüência das relações de colaboração com o setor público é para etapas de finalização e de complementação dos projetos de P\&D (e não para a sugestão e ou iniciação de novos projetos), ter-se-ia uma especificidade para o caso brasileiro.

As atividades de 'Consultoria técnica' vindo em segundo lugar em termos de freqüência, revelando um padrão distinto do observado em estudos em países desenvolvidos (Meyer-Kramer e Schmoch, 1998), vai ao encontro da caracterização do papel da universidade na periferia desenvolvido por Arocena e Sutz (2002). Estes dois relacionamentos mais freqüentes por parte dos grupos de pesquisa refletem a demanda por insumos acadêmicos pouco sofisticados, voltados para pequenas melhorias ou adaptaçốes em concordância com estudos de casos realizados. (Brisolla et. al., 1997). Ademais, a supremacia das atividades de 'Transferência de Tecnologia' $(7,81 \%)$ sobre as de 'Pesquisa científica de uso imediato' $(5,55 \%)$ mostra a primazia de absorção passiva por parte da indústria. ${ }^{12}$

O segundo bloco da Tabela 2 traz a freqüência dos relacionamentos procedentes das empresas para os grupos de pesquisa. Dentre estes, as maiores participações são as de 'Treinamento de pessoal' de 'Engenharia não rotineira' (inclusive o desenvolvimento/fabricação de equipamentos para o grupo) com, respectivamente, 9,86\% e 8,68\% do total dos relacionamentos. Neste bloco, 'Engenharias e Ciência da Computação' abrangem mais da metade dos relacionamentos. Os relacionamentos referentes a 'Treinamento de pessoal' possivelmente compreendem estágios fornecidos a membros dos grupos de pesquisa. Em termos gerais, o 'Desenvolvimento de software' é a atividade de colaboração menos freqüente em ambos os blocos.

\subsection{Articulaçôes Área do Conbecimento e Setor de Atividade}

Esta seção investiga as articulações entre as áreas do conhecimento dos grupos de pesquisa e o setor de atividade das empresas com as quais os líderes dos grupos de pesquisa declararam ter algum tipo de relacionamento, e consiste na tentativa de

12 A exceção a este padrão ocorre somente nas Ciências Biológicas, podendo ser decorrente de especificidades de algumas áreas, como, por exemplo, as relacionadas à biotecnologia. 
efetuar a comparação com estudos similares realizados da perspectiva das empresas (Klevorick et al., 1995; Cohen et al., 2002) ou das universidades (Schartinger et al, 2002; Meyer-Kramer e Schmoch, 1998). As áreas do conhecimento dos grupos de pesquisa escolhidas foram as de maior participação relativa em interações com empresas, ${ }^{13}$ e os setores de atividade selecionados foram os de maior representatividade vinculados às empresas privadas e às empresas pública ou de economia mista.

A Tabela 3 sumaria a articulação entre os setores de atividades das empresas e as respectivas áreas do conhecimento dos grupos de pesquisa que interagiram. O primeiro número refere-se ao total de grupos de pesquisa e o segundo ao total de empresas, nas respectivas áreas do conhecimento e setor de atividade. A magnitude do total dos grupos e das empresas é utilizada como aproximação das interações existentes, visto que as informações foram obtidas no CNPq. A soma das variáveis está sujeita a dupla contagem, devendo as análises ser realizadas somente em cada linha ou coluna.

Uma primeira informação passível de exploração diz respeito ao impacto das áreas do conhecimento nos setores de atividade, ou seja, quais áreas estariam tendo aplicabilidade para um maior número de setores. Neste sentido, em primeiro lugar estão as áreas de Engenharias e, em segundo, as de Química e Ciência da Computação. Por outro lado, algumas áreas contribuem de forma particular para setores específicos, como é o caso de Zootecnia, Medicina e Engenharia de Minas

Cohen et al. (2002) encontraram nos EUA como áreas de maior aplicabilidade setorial os de Ciência da Computação e Ciência dos Materiais. Na base de dados aqui utilizada, apesar destas áreas figurarem como importantes para um substancial número de setores, as de maior aplicabilidade setorial foram Engenharia de Produção e Engenharia Mecânica. Esta configuração, distinta da norte-americana, que é calcada no desenvolvimento de tecnologias de fronteira, se coaduna com o modelo nacional de capacitação e geração interna de tecnologia, baseado em adaptações e em pequenas melhorias incrementais. Por outro lado, o desempenho da Química, mais próximo do padrão observado nos EUA, remete à presença de uma estrutura industrial robusta em química e petroquímica, inclusive com atividades de P\&D compatíveis com os padrões internacionais.

Em termos de articulações específicas, merece destaque a Engenharia Elétrica, com o setor de 'Fabricação de material eletrônico e de aparelhos e equipamentos de comunicações'; a Engenharia de Materiais e Metalúrgica, com 'Metalurgia básica' e 'Fabricação de máquinas de equipamentos'; a Engenharia Mecânica e 'Fabricação de máquinas de equipamentos', Ciência da Computação e 'Atividades de informática e conexas'; Geociências e 'Indústrias Extrativas'; Recursos Florestais e Engenharia

13 Em termos de total de relacionamentos. 
Florestal e 'Fabricação de celulose, papel e produtos do papel'/'Fabricação de produtos de madeira'; e Ecologia e 'Produção e distribuição de energia elétrica'. Observa-se o baixo predomínio de setor(es) ligados à atividade em Física, tendo sua aplicabilidade dispersa entre empresas de distintos setores.

Da perspectiva setorial, na indústria de transformação as indústrias química e petrolífera foram as que mantiveram relacionamentos com a maior diversidade de grupos de pesquisa em termos das áreas preponderantes. ${ }^{14}$ Enquanto que tal desempenho vai ao encontro do identificado em estudos sobre a forte dependência de descobertas científicas, caracterizando-se como setores science-based (Narin et al., 1997; Klevorick et al., 1995; Mansfield, 1991), no País adiciona-se ao mesmo um caráter multidisciplinar das áreas científicas envolvidas nos avanços tecnológicos. As empresas do setor de 'Fabricação de Produtos Químicos' interagiram significativamente com grupos de pesquisa das áreas de Química, Engenharia Química, Medicina Veterinária, Engenharia de Materiais e Metalúrgica e Agronomia, e as empresas do setor de 'Fabricação de coque, álcool, combustíveis nucleares e refino de petróleo' ${ }^{15}$ com grupos de Engenharia Química, Geociências, Engenharia de Materiais e Metalúrgica, Engenharia Mecânica e Química.

Estes resultados apontam, primeiramente, para particularidades específicas, onde existe grande complementaridade entre o conhecimento desenvolvido na academia e a aplicação industrial, podendo-se inclusive 'emprestar' o conceito de "divisão do trabalho" (Schartinger et al., 2001), sendo alguns exemplos Engenharia civil e 'Construção'; Engenharia Florestal e Recursos Florestais' e 'Fabricação de celulose, papel e produtos de papel'. Em segundo lugar, confirmam as características inerentes ao SNI brasileiro baseado em inovações incrementais, adaptativas e de pouca sofisticação, revelado na grande dispersão (ou disseminação) e importância das áreas de Engenharia de Materiais e Metalúrgica e Engenharia de Produção. Portanto, a estrutura industrial e a demanda por conhecimentos científicos e tecnológicos determinam, em grande extensão, o aproveitamento da base de conhecimento existente. As particularidades e características da constituição do SNI são fundamentais na investigação desta dimensão das articulações e da contribuição da infra-estrutura científica nacional nos avanços técnicos e tecnológicos nas empresas.

14 Resultados compatíveis com a PINTEC 2000, na qual 46\% das empresas de Fabricação de Produtos Químicos declararam cooperar com universidades e instituições de pesquisa durante o processo de inovação.

15 Este setor se caracteriza pelo pequeno número de empresas (apenas 16), de forma que a interatividade é expressa na magnitude dos grupos de pesquisa. 


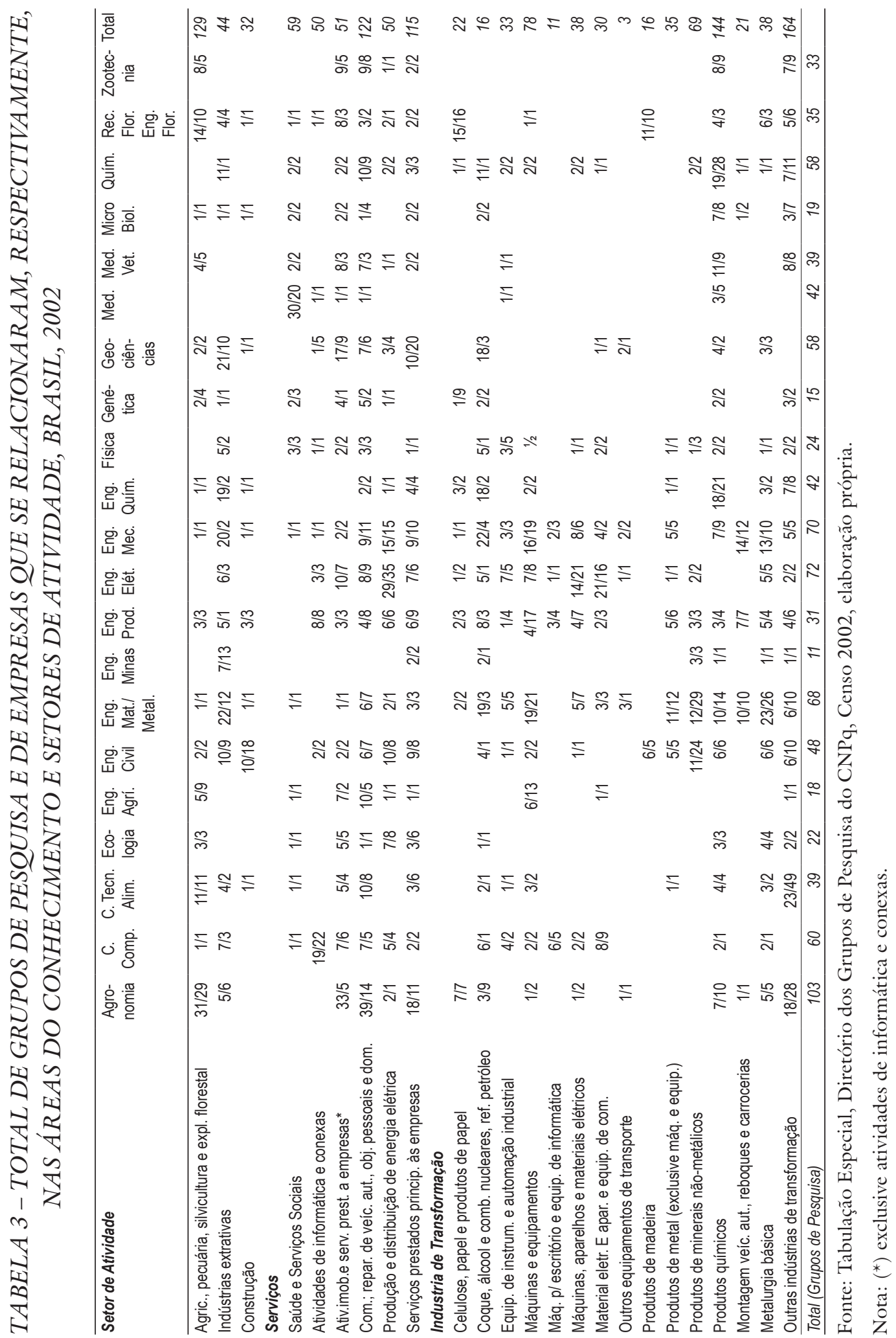




\section{CONCLUSÃO}

A investigação da base de dados do Diretório dos Grupos de Pesquisa do CNPq fornece algumas evidências acerca da articulação recente entre universidades e empresas no País, mesmo que contemplando uma pequena parcela da comunidade científica nacional. Dentre os resultados discorridos ao longo do texto destaca-se a predominância dos fluxos de conhecimentos e serviços oriundos dos grupos de pesquisa para as empresas, sendo os mesmos voltados para atividades rotineiras, de pouca complexidade e sofisticação (consultoria técnica, engenharia não-rotineira e treinamento de pessoal).

As áreas do conhecimento com maior proporção de relacionamentos - Engenharias e Ciência da Computação e Ciências Agrárias - são compatíveis com o modelo de industrialização brasileiro e com incentivos públicos específicos de desenvolvimento setorial. Por sua vez, não é identificada qualquer associação entre a capacitação científica e a colaboração com empresas, como exemplificado pelas áreas de Ciências Biológicas e da Saúde, nas quais o País tem, inclusive, vantagem comparativa em termos internacionais. Nestes casos, estímulos que levem a uma maior atuação do setor privado são necessários para o aproveitamento das oportunidades existentes.

Este panorama se por um lado vai ao encontro do desenvolvido por Cassiolato et al. (1996), sendo inclusive evidência do pouco aproveitamento pelo setor produtivo do conjunto de oportunidades tecnológicas oferecidas pela infra-estrutura de C\&T nacional (p.142), por outro, ressalta o engajamento do sistema público de pesquisa também em pesquisas aplicadas, como encontrado por Schwartzman (1989). As universidades e instituições de pesquisa brasileiras são potencialmente capazes de contribuir para a solução de problemas técnicos e em avanços tecnológicos na indústria (Quental et al., 2000), sendo importante não apenas a existência de mecanismos efetivos e eficientes de interação com o setor produtivo, mas também a demanda pelos mesmos nos últimos.

A instabilidade macroeconômica e os impactos sofridos com a abertura dos anos 1990 levaram à adoção de novas estratégias de sobrevivência por parte das empresas. Distintamente do almejado, a adequação das empresas ao novo cenário deu-se primordialmente na aquisição de insumos tangíveis (máquinas e equipamentos) em detrimento da aquisição e/ou desenvolvimento de insumos intangíveis. (Cassiolato e Szapiro, 2003). A interação com universidades e instituiçóes de pesquisa pode ser considerada como um mecanismo capaz de fomentar a aquisição de insumos intangíveis fundamentais para a geração e desenvolvimento de tecnologias internamente. 
Espera-se com este trabalho ter explorado as potencialidades da base de dados em questão como mecanismo auxiliar na investigação das interações universidade-empresa e área do conhecimento-setor de atividade. As limitações inerentes à coleta das informaçốes e à metodologia adotada devem estar sempre em mente, devendo os resultados ser interpretados com precaução, ou seja, como uma aproximação. Esta base de dados pode ser explorada de inúmeras outras formas como, por exemplo, em pesquisas no nível de Unidades da Federação. ${ }^{16}$ A continuidade de esforços semelhantes por parte do CNPq talvez permita, no futuro, comparações intertemporais, concomitantemente com o aumento do nosso entendimento sobre o tema no País.

Por fim, como agenda de pesquisa para o futuro propóe-se pelo menos três desdobramentos a partir do presente trabalho. Um primeiro consiste na combinação destas informações com estudos de casos a serem realizados, que complementem a investigação do tema da cooperação entre universidades e empresas no País. Um segundo, consiste no levantamento das produções científica e tecnológica dos grupos de pesquisa que se relacionaram com empresas na compreensão de possíveis desdobramentos e causalidades (semelhante a investigações realizadas na literatura por Stephan, 2001 e Ranga, et. al., 1999). E em terceiro, o desenvolvimento e concepção de indicadores voltados para as interações universidade-indústria, no âmbito de um escopo maior de indicadores de C\&T para países em desenvolvimento.

\section{BIBLIOGRAFIA}

Acts, Z. J; Audretsch, D. B; Feldman, M. P. R\&D spillovers and recipient firm size. The American Economic Review, v. 82, n. 1, p. 363-367, March 1994.

Arocena, R.; Sutz, J. Knowledge, innovation and learning: systems and policies in the north and in the south. In: Cassiolato, J. E; Lastres, H. M. M; Maciel, M. L. (orgs.), Systems of innovation and development - evidence from Brazil. $1^{\mathrm{a}} \mathrm{ed}$. Massachusetts: Edward Elgar, 2003, Capitulo 11, p. 291-310.

Audretsch, D. B.; Stephan, P. E. Company - scientist locational links: the case of biotechnology. The American Economic Review, v. 86, n. 3, p. 641-652, June 1996.

Beise, M.; Stahl, H. Public research and industrial innovations in Germany. Research Policy, v. 28, n. 4, p. 397-422, abril 1999.

Breschi, S.; Lissoni, F. Knowledge spillovers and local innovation systems: a critical survey. Industrial and Corporate Change, v. 10, n. 4, p. 975-1005, 2001.

Brisolla, S.; Corder, S.; Gomes, E.; Mello, D. As relações universidade-empresagoverno: um estudo sobre a Universidade Estadual de Campinas. Educação \& Sociedade, ano XVIII, n. 61, p. 187-209, dezembro 1997.

16 Ver Rapini e Campos (2004) para investigação sobre Minas Gerais. 
Carneiro, S. J.; Lourenço, R. Pós-graduação e pesquisa na universidade. In: Viotti, E. B.; Macedo, M. (orgs.), Indicadores de ciência, tecnologia e inovação no Brasil. Campinas: Editora da Unicamp, 2003, Capítulo 4, p.169-227.

Cassiolato, J. E.; Szapiro, M. Alguns indicadores de inovação no Brasil: os dados da PINTEC e a importação de tecnologia. In: Cassiolato, J. E.; Lastres, M. M. H. (orgs.), Novas politicas de inovação: lições para o Brasil. IE/URFJ, 2003. Mimeografado.

Cassiolato, J. E.; Albuquerque, E. As especificidades do sistema de inovação do setor saúde: uma resenha da literatura como introdução a uma discussão sobre o caso brasileiro. Estudos FesSBE I, 2000.

Cassiolato, J. E.; Gadelha, C. G.; Albuquerque, E.; Britto, J. A relação universidade e instituiçôes de pesquisa com o setor industrial: uma análise de seus condicionantes. IE/UFRJ, 1996. Mimeografado.

CNPq. Diretório dos grupos de pesquisa. Disponível em: http://lattes.cnpq.br/censo2002.

Cohen, W. M.; Nelson, R. R.; Walsh, J. P. The influence of public research on industrial R\&D. Management Science, v. 48, n. 1, p. 1-23, January 2002.

Costa, E. F. A interação universidade/empresas e o papel do Estado: um caso de sucesso. Interação Universidade Empresa, Brasília: IBICT, p. 262-281, 1999.

Dahlman, C. J.; Frischtak, C. R. National systems supporting technical advance in industry: the Brazilian experience. In: Nelson, R. (org.), National innovation systems: a comparative analysis. New York, Oxford: Oxford University, 1993, Chapter 13, p. 76-114.

Dorfman, N. S. Route 128: the development of a regional high technology economy. Research Policy, v. 12, n. 6, p. 299-316, December 1989.

Etzkowitz, H. Bridging the gap: the evolution of industry-university links in the United States. In: Branscombs, L. M.; Kodama, F.; Florida, R. (orgs.), Industrializing knowledge - university-industry linkages in Japan and the United States. Cambridge: The MIT Press, 1999, p. 203-233.

Faulkner, W.; Senker, J. Making sense of diversity: public-private sector research linkages in three technologies. Research Policy, v. 23, n. 6, p. 673-695, November 1994.

Feldman, M. The geography of innovation. Netherlands: Kluwer Academic Publishers, 1994.

Frantz, T. R. O papel da interação universidade e empresa na implantação de um programa de capacitação tecnológica em região periférica (A experiência do programa de cooperação científica e tecnológica do noroeste do RS). Interação Universidade Empresa, Brasília: IBICT, v. 2, p. 65-89, 1999..

Godin, B. Research and the practice of publication in industries. Research Policy, v. 25, n. 4, p. 587-606, June 1996. 
Gregolin, J. A. R. É possível aumentar a contribuição social da universidade via interação com empresas? Interação Universidade Empresa, Brasília: IBICT, v. 1, p. 180-203, 1999.

Grupp, H. Spillover effects and science base of innovations reconsidered: an empirical aprroach. Evolutionary Economics, v. 6, p. 175-197, 1996.

Hemais, C. A.; Rosa, E.; Barros, H. M. Patent activities in North América and Brazilian Universities: a comparative study. Third Triple Helix International Conference: The Endless Transition, Rio de Janeiro, 2000.

IBGE. Pesquisa industrial inovação tecnológica 2000. Rio de Janeiro: IBGE, 2002. . Brasil em múmeros, v. 13. Rio de Janeiro: IBGE, 2003.

Jaffe, A. B.; Trajtenberg, M; Henderson, R. Geographic localization of knowledge spillovers as evidence by patent citation. The Quarterly Journal of Economics, v. 108, n. 3, p. 577-598, August 1993.

Jasinski, A. H. New development in science-industry linkages in Poland. Science and Public Policy, v. 24, n. 2, p. 93-99, April 1997.

Klevorick, A. K.; Levin, R.; Nelson, R.; Winter, S. On the sources and significance of inter-industry differences in technological opportunities. Research Policy, v. 24, n. 2, p. 185-205, March 1995.

Mansfield, E. Academic research and industrial innovation. Research Policy, v. 20, n. 1, p. 1-12, February 1991.

Mansfield, E.; Lee, J. The modern university: contributor to industrial innovation and recipient of industrial P\&D support. Research Policy, v. 25, n. 7, p. 1047 1058, October 1996.

McFetridge, D. G. The Canadian system of industrial innovation. In: Nelson, R. (org.), National innovation systems: a comparative analysis. New York, Oxford: Oxford University, 1993, p. 299-323.

Melo, L. C. de Organização do fomento para a promoção da cooperação universidadeempresa: a experiência da Facepe no período 1995-1998. Interação Universidade Empresa, Brasília: IBICT, v. 2, p. 137-149, 1999.

Meyer-Kramer, F; Schmoch, U. Science-based technologies: university- industry interactions in four fields. Research Policy, v. 27, n. 8, p. 835-851, December 1998.

Moravcsik, M. J. The role of science in technology transfer. Research Policy, v. 12, n. 5, p. 287-296, October 1983.

Nelson, R. Capitalism as an engine of progress. Research Policy, v. 19, n. 3, p. 193214, June 1990.

Oyebisi, T. O.; Ilori, M. O.; Nassar; M. L. Industry-academic relations: an assessment of the linkages between a university and some enterprises in Nigeria. Technovation, v. 16, n. 4, p. 203-209, 1996. 
Pavitt, K. Sectoral patterns of technical change: towards a taxonomy and a theory. Research Policy, v. 13, n. 6, p. 343-373, December 1984.

. What makes basic research economically useful. Research Police, v. 20, n. 2, p. 109-119, April 1991.

Quental, C.; Gadelha, C. A.; Fialho, B. de C. Brazilian health innovation system. In: Third Triple Helix International Conference: The Endless Transition, Rio de Janeiro, 2000.

Ranga, L. M.; Debackere, K.; Tunzelmann, N. V. Entrepreneurial universities and the dynamics of academic knowledge production: a case study of basic vs. applied research in Belgium. Scientometrics, v. 58, n. 2, p. 301-320, July 2003.

Rappel, E. Integração universidade-indústria: os "porques" e os “comos". Interação Universidade Empresa, Brasília: IBICT, p. 90-106, 1999.

Rapini, M. S. Interação universidade-indústria no Brasil: uma análise exploratória a partir do Diretório de Pesquisas do CNPq.2004. Dissertação (Mestrado), apresentada ao Instituto de Economia da UFRJ, Rio de Janeiro.

Rapini, M. S; Campos, B.C. As universidades mineiras e suas interações com a indústria: uma análise a partir de dados do Diretório dos Grupos de Pesquisas do Cnpq. In: XI Seminário sobre Economia Mineira, Diamantina, 2004.

Rosenberg, N. Scientific instrumentation and univeristy research.? Research Policy, v. 21, n. 4, p. 381-390, August 1992.

. Why firms do basic research (with their own money)? Research Policy, v. 19, n. 2, p. 165-174, April 1990.

Rosenberg, N.; Nelson, R. American university and technical advance in industry. Research Policy, v. 23, n. 3, p. 323-348, May1994.

Salomão, J. R. A incubação de empresas e projetos cooperativos como mecanismo de interação com a universidade. Interação Universidade Empresa, Brasília: IBICT, v. 2, p. 188-207, 1999.

Salter, A.; Martin, B. The economic benefits of publicly funded basic research: a critical review. Research Policy, v. 30, n. 3, p. 509-532, March 2001.

Schartinger, D.; Shibany, A.; Gassler, H. Interactive relations between universities and firms: empirical evidence for Austria. Journal of Technology Transfer, v. 26, p. 255-268, 2001.

Schartinger, D.; Rammer, C.; Fisher, M. M.; Fröhlich, J. Knowledge interactions between universtities and industry in Austria: sectoral patterns and determinants. Research Policy, v. 31, n. 3, p. 303-328, March 2002.

Schwartzman, S. Coming full circle: a reappraisal of university reform in Latin América. Minerva, v. 24, p. 456-475, 1986.

Schneider, C. A. A transferência de tecnologia entre universidade-indústria na vertente incubação de empresa de base tecnologica. Interação Universidade Empresa, Brasília: IBICT, p.204-212, 1999. 
Silva, L. E.; Mazzali, L. Parceria tecnológica universidade-empresa: um arcabouço conceitual para a análise de gestão dessa relação. Parcerias Estratégicas, n. 11, MCT, 2001.

Stankiewicz, R. Spin-off companies from universities. Science and Public Policy, v. 21, n. 2, p. 99-107, Abril 1994.

Stephan, P. E. Educational implications of university-industry technology transfer. Journal of Technology Transfer, v. 26, p. 199-205, 2001.

Suzigan, W.; Villela, A. V. Industrial policy in Brazil.Campinas: Universidade Estadual de Campinas, Instituto de Economia, 1997.

Sutz, J. The university- industry-government relations in Latin América. Research Policy, v. 29, n. 2, p. 279-290, February 2000.

Szapiro, M. Dowgrading local capabilities in IT: the telecom innovation system in Campinas. In: Cassiolato, J. E; Lastres, H. M. M; Maciel, M. L. (orgs.), Systems of innovation and development - evidence from Brazil. $\mathrm{I}^{\mathrm{a}}$ ed. Massachusetts: Edward Elgar, 2003, Capítulo 19, p. 470-498.

Theis, I. M. Inovação, desenvolvimento regional e parques tecnológicos: uma análise crítica do caso brasileiro. In: Fisher, T. (org.), Gestão do desenvolvimento e poderes locais: marcos teóricos e avaliação. Salvador: Casa da Qualidade, 2002.

Varga, A. Regional economic effects of university research: a survey. Working Paper, Viena: Department for Economics Geography and Geoinformatics, University of Economics and Business Administration, 1997.

Vargas, M. A.; Filho, N. S.; Alievi, R. M. Sistema gaúcho de inovação: avaliação de arranjos locais selecionados. In: Cassiolato, J. E.; Lastres, H. M. M. (orgs.), Globalização e inovação localizada: experiências de sistemas locais no Mercosul. Brasília: IBICT/MCT, 1999, Capítulo 12, p. CC.

Velho, L. Relações universidade-empresa: desvelando mitos. Campinas, SP: Autores Associados, Coleção educação contemporânea, 1996. 